\title{
Submicrosecond Q-Switching Er-Doped All-Fiber Ring Laser Based on Black Phosphorus
}

\author{
Yao Cai, Yanliang He, Xiaoke Zhang, Rui Jiang, Chenliang Su, and Ying Li \\ SZU-NUS Collaborative Innovation Center for Optoelectronic Science \& Technology, \\ Key Laboratory of Optoelectronic Devices and Systems of Ministry of Education and Guangdong Province, \\ College of Optoelectronic Engineering, Shenzhen University, Shenzhen 518060, China \\ Correspondence should be addressed to Ying Li; queenly@szu.edu.cn
}

Received 30 June 2017; Accepted 29 August 2017; Published 4 October 2017

Academic Editor: Yan Luo

Copyright (C) 2017 Yao Cai et al. This is an open access article distributed under the Creative Commons Attribution License, which permits unrestricted use, distribution, and reproduction in any medium, provided the original work is properly cited.

\begin{abstract}
Black phosphorus (BP), a new two-dimensional (2D) material, has been deeply developed for extensive applications in electronics and optoelectronics due to its similar physical structure to graphene and thickness dependent direct band gap. Here, we demonstrated a submicrosecond passive Q-switching Er-doped fiber laser with BP as saturable absorber (SA). The BP saturable absorber was fabricated by mechanical exfoliation method. By taking full advantage of the ultrafast relaxation time of BP-SA and careful design of compact ring cavity, we obtained stable Q-switching pulses output with a shortest duration as narrow as $742 \mathrm{~ns}$. With increasing the pump power, the pulse repetition rate accreted gradually almost linearly from 9.78 to $61.25 \mathrm{kHz}$, and the pulse duration declined rapidly at lower pump power regime and retained approximate stationary at higher pump power regime from 3.05 to $0.742 \mu \mathrm{s}$. The experimental results indicate that BP-SA can be an effective SA for nanosecond Q-switching pulse generation.
\end{abstract}

\section{Introduction}

Pulsed fiber lasers have been widely applied in various applications ranging within machining, material processing, environmental sensing, medicine, laser processing, fiber sensor, and long-range optical communication. The most common pulse generation methods used in fiber laser include Q-switching and mode-locking techniques [1-6]. There are two types of Q-switching approaches: active and passive one. Among them, passive Q-switching technology based on saturable absorber (SA) has made remarkable progress in view of compact, low cost, flexible, and so on. Since the Nd:glass (the first generation of SA) was successfully used for pulse generation in 1966 [7], a wide variety of SAs have been intensively developed, such as Semiconductor Saturable Absorption Mirrors (SESAMs) [8, 9], Carbon Nanotubes (CNTs) [1013], graphene [14-18], Topological Insulator (TI) [19, 20], and Transition Metal Dichalcogenides (TMDs) [21-24]. The SESAMs are utilized in most of commercially available laser systems for high flexibility and stability. However, SESAMs have relatively narrow operation bandwidth and require complex fabrication and packaging [1]. Recently, the research on broadband SAs based on CNT or graphene has presented explosive development for broad operation bandwidth, ultrafast recovery times, low saturation intensity, low cost, and easy fabrication [10-18]. Nevertheless, they still have some drawbacks. The spectral response range of CNTs sensitively depends on their diameter and chirality, restricting their practical applications in specific wavelength or broadband tenability [13]. And, graphene has relatively weak optical absorption ( 2.3\%/layer [20]) due to its gapless band structure, which limits its application in fiber laser. Another 2D material, transition metal dichalcogenides (TMDs) $\left(\mathrm{MoS}_{2}\right.$ [21], $\mathrm{WS}_{2}[22], \mathrm{MoSe}_{2}[23,24]$, etc.) has been developed as saturable absorber with high performances [21]. Although they have higher optical absorption at specific wavelength, the optical response mainly locates at visible region due to their comparatively large band gap $\left(\sim 1.8 \mathrm{eV}\right.$ for $\mathrm{MoS}_{2}, \sim 2.1 \mathrm{eV}$ for $\mathrm{WS}_{2}$, and $\sim 1.7 \mathrm{eV}$ for $\left.\mathrm{MoSe}_{2}\right)[25,26]$.

Very recently, another $2 \mathrm{D}$ material, black phosphorus (BP), has been rediscovered for various applications in electronics and optoelectronics [27]. Its structure is similar to graphene, in which individual atomic layers stacked together by van der Waals interaction. BP has layer-dependent direct 


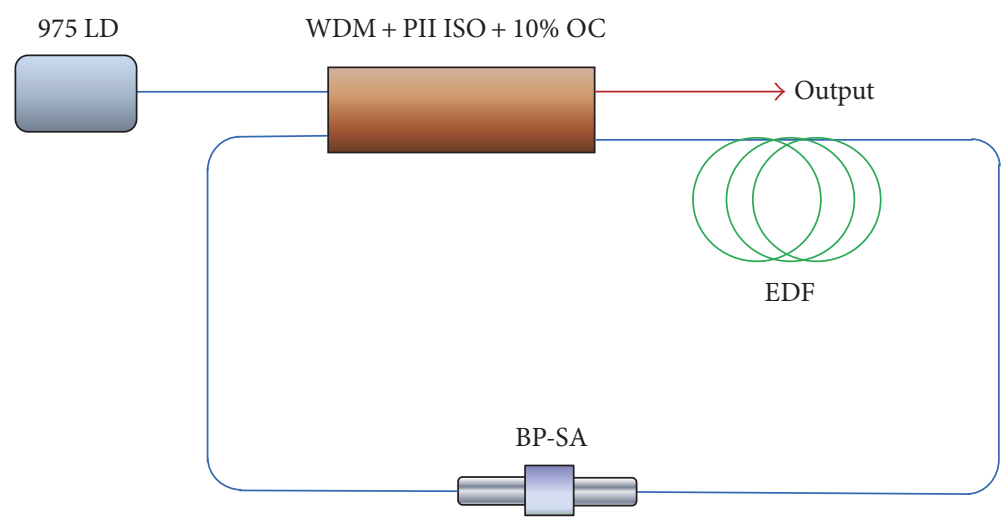

(a)

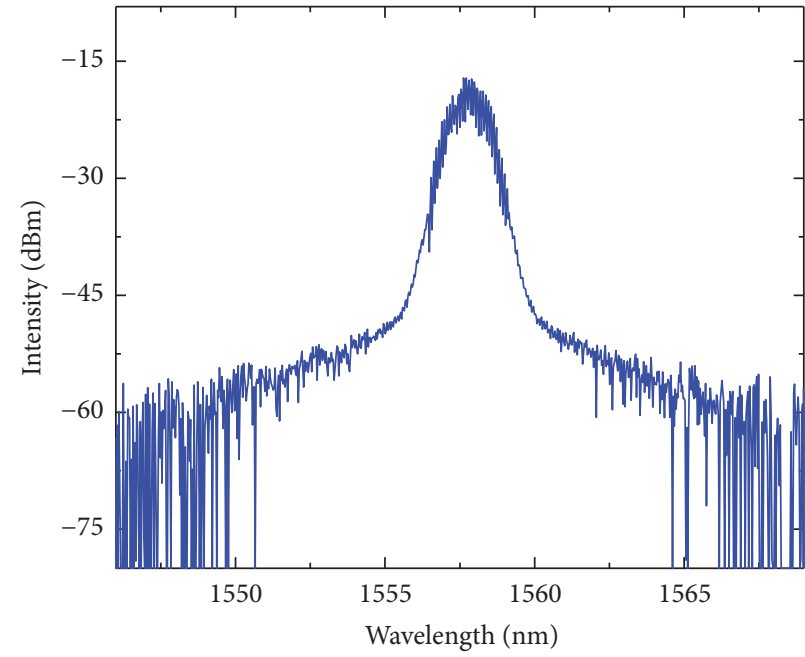

(b)

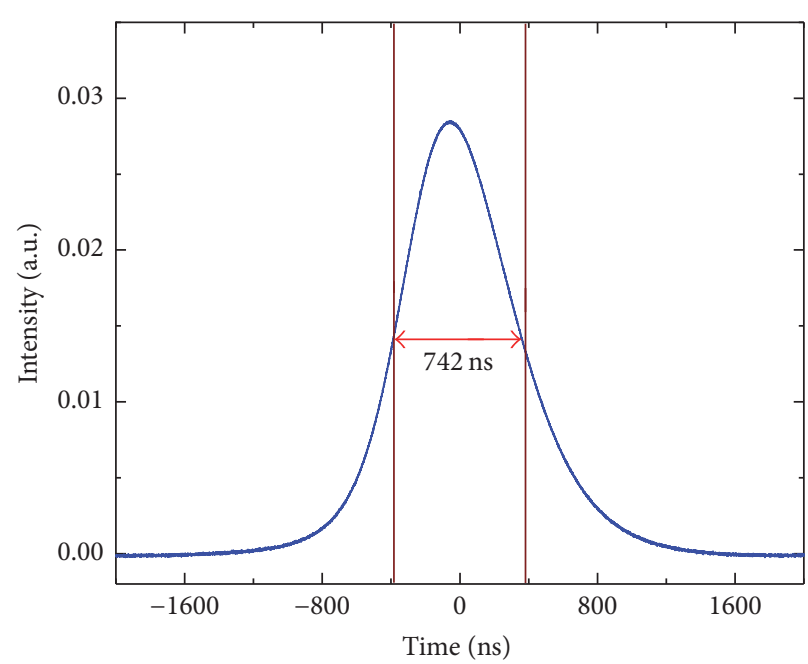

(c)

FIgURE 1: (a) Schematic of Er-doped black phosphorus-based Q-switching fiber laser. (b) Typical Q-switched output optical spectrum. (c) The minimum duration pulse obtained in our fiber laser.

electronic band gap, which can be tuned from $\sim 0.3$ (bulk) to $\sim 2 \mathrm{eV}$ (monolayer) [28], covering nearly whole near and mid-infrared wavelength. Therefore BP can be regarded as a significant benefit material for ultrafast photonics and high frequency optoelectronics in infrared wavelength. Lately, researches have paid close attention to its nonlinear optical response and used it as SA in lasers [29-31]. Even [32] shows the recovery time of BP is as short as $26 \mathrm{fs}$ (much shorter than other SAs mentioned above), revealing its tremendous potential in ultrafast pulse generation.

In this paper, we also focus on the capacity of BP in ultrashort pulse generation and demonstrate a submicrosecond Q-switching pulse with a compact Er-doped all-fiber ring laser. The BP material was prepared by mechanical exfoliation method and as an effective SA for short Q-switching pulse generation. After inserting this BP-SA into a well design compact Er-doped fiber laser, we achieved stable Q-switching pulse output. The narrowest Q-switching pulse we obtained is as short as $742 \mathrm{~ns}$. The experimental results show the BP's potential for narrow $\mathrm{Q}$-switching pulse generation.

\section{Experimental Setup and Results}

The black phosphorus-based SA was prepared by mechanical exfoliation method, whose details have been shown in [30]. To achieve a submicrosecond Q-switched pulse, ultrashort laser cavity should be employed. We designed a special compact all-fiber ring cavity shown in Figure 1(a) with an optical integrated device for the joint function of wavelengthdivision multiplexer (WDM), polarization-insensitive isolator (PI-ISO), and $10 \%$ output coupler (OC). The total cavity length is $4.7 \mathrm{~m}$ with $0.95 \mathrm{~m}$ highly doped erbium-doped fiber (EDF, LIEKKI Er 80-8/125) as gain medium. Others are standard single-mode fiber (SMF-28). The laser is pumped by a $975 \mathrm{~nm}$ laser diode (LD) with maximum power of $500 \mathrm{~mW}$. The BP-SA is incorporated into the cavity between the EDF and integrated device. There is not any extra component in this fiber laser. The output is characterized by an optical spectrum analyzer (Ando AQ-6317B) and a real-time oscilloscope with bandwidth of $4 \mathrm{GHz}$ (Agilent Technol., DSO9404A) combined with a $5 \mathrm{GHz}$ photodetector (Thorlabs SIR5). 


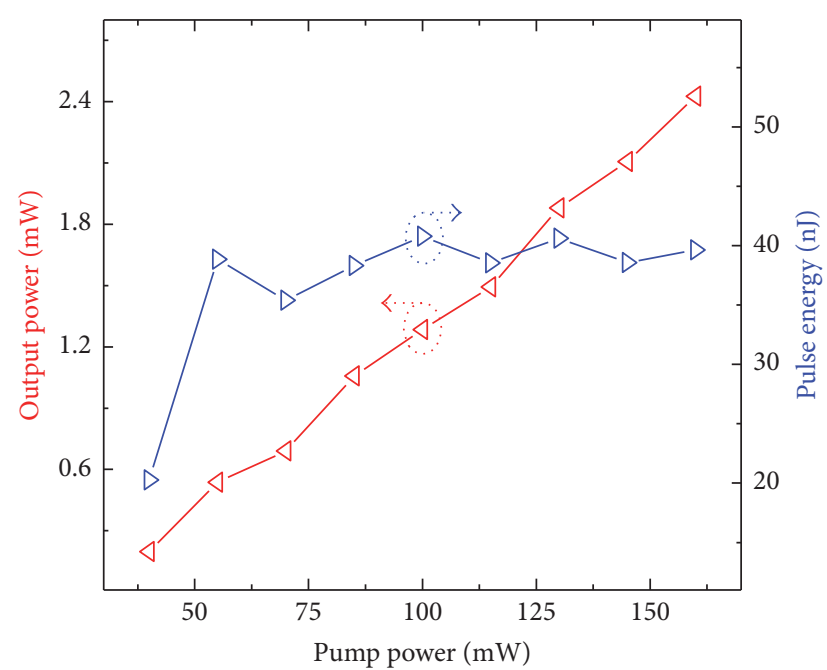

$\triangleleft-$ Output power

$\rightarrow-$ Pulse energy

(a)

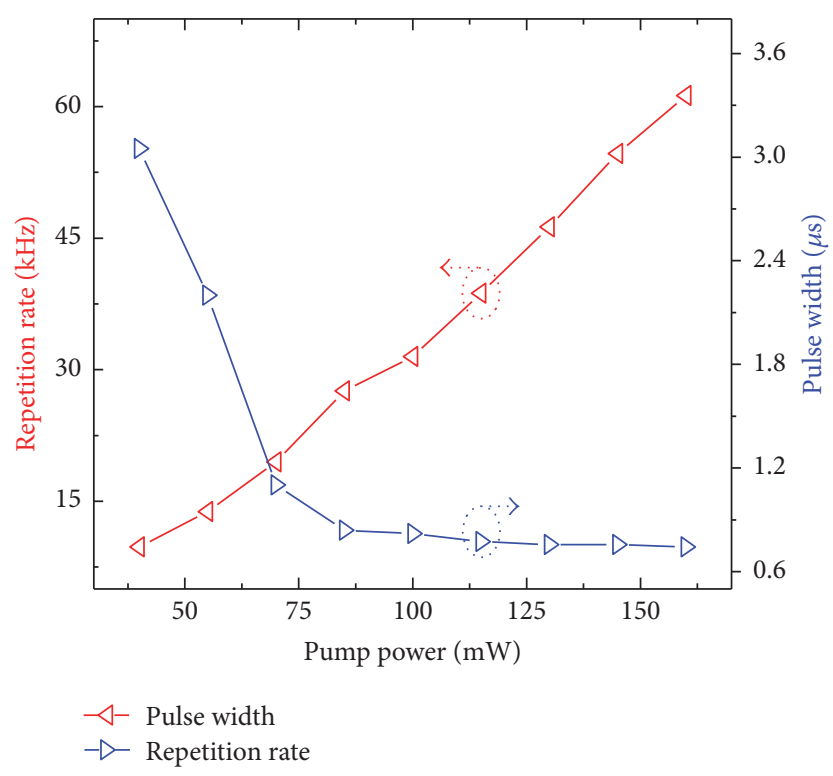

(b)

FIGURE 2: The evolution of output average power and pulse energy (a) and pulse repetition rate and duration versus incident pump power (b) as pump power increases.

Without the BP-SA device, the central wavelength is located at $1600 \mathrm{~nm}$, suggesting that the optical integrated device possesses low insertion loss. In this condition, we cannot obtain Q-switching operation state no matter how we adjust pump power and apply stress on fiber (correspondingly changing fiber birefringence to control the polarization rate of light in cavity). After the BP-SA device is introduced into the cavity, stable Q-switching output can be obtained once the pump power exceeds $40 \mathrm{~mW}$. Figure 1(b) shows typical Q-switching spectrum with slight modulation. The central wavelength is $1557.9 \mathrm{~nm}$. With increasing pump power, the durations of Q-switching pulse decrease gradually. At max revealable pump power of $160 \mathrm{~mW}$, we obtained minimum pulse width as narrow as $742 \mathrm{~ns}$ as shown in Figure 1(c). It is worth noting that, keeping increasing pump power above $160 \mathrm{~mW}$, the Q-switching state becomes unstable and disappears ultimately.

In addition, we also measured the evolution process of pulses with the pump power increasing to confirm the Qswitching operating state, shown in Figure 2. As it can be seen, with pump power increasing from 40 to $160 \mathrm{~mW}$, the average output power increases almost linearly from $198 \mu \mathrm{W}$ to $2.43 \mathrm{~mW}$, but the pulse energy fluctuates between 20.2 and $40.8 \mathrm{~nJ}$. Simultaneously, the pulse repetition rate accretes gradually also almost linearly from 9.78 to $61.25 \mathrm{kHz}$, and the pulse duration declines rapidly at lower pump power regime and keeps approximately unchanged at higher power regime from 3.05 to $0.742 \mu$ s. These entire matches with typical Qswitching state confirm its operating state.

\section{Conclusions}

In conclusion, we focus on the capacity of BP in ultrashort pulse generation and demonstrate a submicrosecond
Q-switching pulse with a compact Er-doped all-fiber ring laser. The BP material was prepared by mechanical exfoliation method and as an effective SA for short Q-switching pulse generation. It has narrow Q-switching pulse as short as $742 \mathrm{~ns}$. With increasing the pump power, the pulse repetition rate accreted gradually almost linearly from 9.78 to $61.25 \mathrm{kHz}$, and the pulse duration declined rapidly at lower pump power regime and retained approximate stationary at higher pump power regime from 3.05 to $0.742 \mu \mathrm{s}$. The experimental results indicate that BP-SA can be an effective SA for nanosecond Q-switching pulse generation.

\section{Conflicts of Interest}

The authors declare that there are no conflicts of interest regarding the publication of this paper.

\section{Acknowledgments}

The authors would like to acknowledge supports from the National Natural Science Foundation of China (Grant nos. 61575127, 61505122, and 61505117), the Natural Science Foundation of Guangdong Province (Grant nos. 2016A030310065 and 2014A030310279), the Science and Technology Planning Project of Guangdong Province (Grant no. 2016B050501005), and the Educational Commission of Guangdong Province (Grant nos. 2016KCXTD006 and 2015KQNCX146).

\section{References}

[1] U. Keller, "Recent developments in compact ultrafast lasers," Nature, vol. 424, no. 6950, pp. 831-838, 2003.

[2] V. V. Dvoyrin, V. M. Mashinsky, and E. M. Dianov, "Yb - Bi pulsed fiber lasers," Optics Letters, vol. 32, no. 5, pp. 451-453, 2007. 
[3] P. Dupriez, A. Piper, A. Malinowski et al., "High average power, high repetition rate, picosecond pulsed fiber master oscillator power amplifier source seeded by a gain-switched laser diode at 1060 nm," IEEE Photonics Technology Letters, vol. 18, no. 9, pp. 1013-1015, 2006.

[4] F. X. Kärtner, J. Aus der Au, and U. Keller, "Mode-locking with slow and fast saturable absorbers-what's the difference?" IEEE Journal on Selected Topics in Quantum Electronics, vol. 4, no. 2, pp. 159-168, 1998.

[5] H. L. Offerhaus, N. G. Broderick, D. J. Richardson, R. Sammut, J. Caplen, and L. Dong, "High-energy single-transverse-mode Q-switched fiber laser based on a multimode large-mode-area erbium-doped fiber," Optics Letters, vol. 23, no. 21, pp. 1683$1685,1998$.

[6] B. Ding, Y. Cheng, J. Wu et al., "A unique multifunctional cluster-based nano-porous Terbium organic material: real-time detection of benzaldehyde, visually luminescent sensor for nitrite and selective high capacity capture of Congo Red," Dyes and Pigments, vol. 146, pp. 455-466, 2017.

[7] D. A. Stetser and A. J. Demaria, "Optical spectra of ultrashort optical pulses generated by mode-locked glass:nd lasers," Applied Physics Letters, vol. 9, no. 3, pp. 118-120, 1966.

[8] U. Keller, K. J. Weingarten, F. X. Kärtner et al., "Semiconductor saturable absorber mirrors (SESAM's) for femtosecond to nanosecond pulse generation in solid-state lasers," IEEE Journal on Selected Topics in Quantum Electronics, vol. 2, no. 3, pp. 435451, 1996.

[9] J. F. Li, H. Y. Luo, Y. L. He et al., "Semiconductor saturable absorber mirror passively Q-switched $2.97 \mu \mathrm{m}$ fluoride fiber laser," Laser Physics Letters, vol. 11, no. 6, Article ID 065102, 2014.

[10] S. W. Harun, M. A. Ismail, F. Ahmad et al., "A Q-switched erbium-doped fiber laser with a carbon nanotube based saturable absorber," Chinese Physics Letters, vol. 29, no. 11, Article ID 114202, 2012.

[11] B. Dong, J. Hao, J. Hu, and C.-Y. Liaw, "Short linear-cavity Q-switched fiber laser with a compact short carbon nanotube based saturable absorber," Optical Fiber Technology, vol. 17, no. 2, pp. 105-107, 2011.

[12] K. Kieu and F. W. Wise, "Soliton thulium-doped fiber laser with carbon nanotube saturable absorber," IEEE Photonics Technology Letters, vol. 21, no. 3, pp. 128-130, 2009.

[13] D.-P. Zhou, L. Wei, B. Dong, and W.-K. Liu, "Tunable passively Q-switched erbium-doped fiber laser with carbon nanotubes as a saturable absorber," IEEE Photonics Technology Letters, vol. 22, no. 1, pp. 9-11, 2010.

[14] W.-J. Cao, H.-Y. Wang, A.-P. Luo, Z.-C. Luo, and W.-C. $\mathrm{Xu}$, "Graphene-based, $50 \mathrm{~nm}$ wide-band tunable passively Qswitched fiber laser," Laser Physics Letters, vol. 9, no. 1, pp. 54-58, 2012.

[15] L. Q. Zhang, Z. Zhuo, J. X. Wang, and Y. Z. Wang, "Passively Q-switched fiber laser based on graphene saturable absorber," Laser Physics, vol. 22, no. 2, pp. 433-436, 2012.

[16] Z. T. Wang, Y. Chen, C. J. Zhao, H. Zhang, and S. C. Wen, "Switchable dual-wavelength synchronously Q-switched erbium-doped fiber laser based on graphene saturable absorber," IEEE Photonics Journal, vol. 4, no. 3, pp. 869-876, 2012.

[17] H. Ahmad, F. D. Muhammad, M. Z. Zulkifli, and S. W. Harun, "Graphene-oxide-based saturable absorber for all-fiber Q-switching with a simple optical deposition technique," IEEE Photonics Journal, vol. 4, no. 6, pp. 2205-2213, 2012.
[18] Q. Bao, H. Zhang, Y. Wang et al., "Atomic-layer craphene as a saturable absorber for ultrafast pulsed lasers," Advanced Functional Materials, vol. 19, no. 19, pp. 3077-3083, 2009.

[19] C. Zhao, H. Zhang, and X. Qi, "Ultra-short pulse generation by a topological insulator based saturable absorber," Applied Physics Letters, vol. 101, no. 21, Article ID 211106, 2012.

[20] S. Chen, L. Miao, X. Chen et al., "Few-layer topological insulator for all-optical signal processing using the nonlinear kerr effect," Advanced Optical Materials, vol. 3, no. 12, pp. 1769-1778, 2015.

[21] H. Zhang, S. B. Lu, J. Zheng et al., "Molybdenum disulfide (MoS2) as a broadband saturable absorber for ultra-fast photonics," Optics Express, vol. 22, no. 6, pp. 7249-7260, 2014.

[22] M. Zhang, G. Hu, G. Hu et al., "Yb- and Er-doped fiber laser Qswitched with an optically uniform, broadband WS2 saturable absorber," Scientific Reports, vol. 5, Article ID 17482, 2015.

[23] B. Chen, X. Zhang, K. Wu, H. Wang, J. Wang, and J. Chen, "Qswitched fiber laser based on transition metal dichalcogenides MoS2, MoSe2, WS2, and WSe2," Optics Express, vol. 23, no. 20, pp. 26723-26737, 2015.

[24] R. I. Woodward, R. C. T. Howe, T. H. Runcorn et al., "Wideband saturable absorption in few-layer molybdenum diselenide (MoSe2) for Q-switching Yb-, Er- and Tm-doped fiber lasers," Optics Express, vol. 23, no. 15, pp. 20051-20061, 2015.

[25] F. Bonaccorso and Z. Sun, "Solution processing of graphene, topological insulators and other $2 \mathrm{~d}$ crystals for ultrafast photonics," Optical Materials Express, vol. 4, no. 1, pp. 63-78, 2014.

[26] Q. H. Wang, K. Kalantar-Zadeh, A. Kis, J. N. Coleman, and M. S. Strano, "Electronics and optoelectronics of two-dimensional transition metal dichalcogenides," Nature Nanotechnology, vol. 7, no. 11, pp. 699-712, 2012.

[27] F. Xia, H. Wang, and Y. Jia, "Rediscovering black phosphorus as an anisotropic layered material for optoelectronics and electronics," Nature Communications, vol. 5, article 4458, 2014.

[28] L. Li, Y. Yu, G. J. Ye et al., "Black phosphorus field-effect transistors," Nature Nanotechnology, vol. 9, no. 5, pp. 372-377, 2014.

[29] Y. Chen, G. Jiang, S. Chen et al., "Mechanically exfoliated black phosphorus as a new saturable absorber for both Q-switching and mode-locking laser operation," Optics Express, vol. 23, no. 10, pp. 12823-12833, 2015.

[30] J. Sotor, G. Sobon, W. Macherzynski, P. Paletko, and K. M. Abramski, "Black phosphorus saturable absorber for ultrashort pulse generation," Applied Physics Letters, vol. 107, no. 5, Article ID 051108, 2015.

[31] Z.-C. Luo, M. Liu, Z.-N. Guo et al., "Microfiber-based few-layer black phosphorus saturable absorber for ultra-fast fiber laser," Optics Express, vol. 23, no. 15, pp. 20030-20039, 2015.

[32] H. Mu, S. Lin, Z. Wang et al., "Black phosphorus-polymer composites for pulsed lasers. Advanced Optical Materials," Advanced Optical Materials, vol. 3, no. 10, pp. 1447-1453, 2015. 

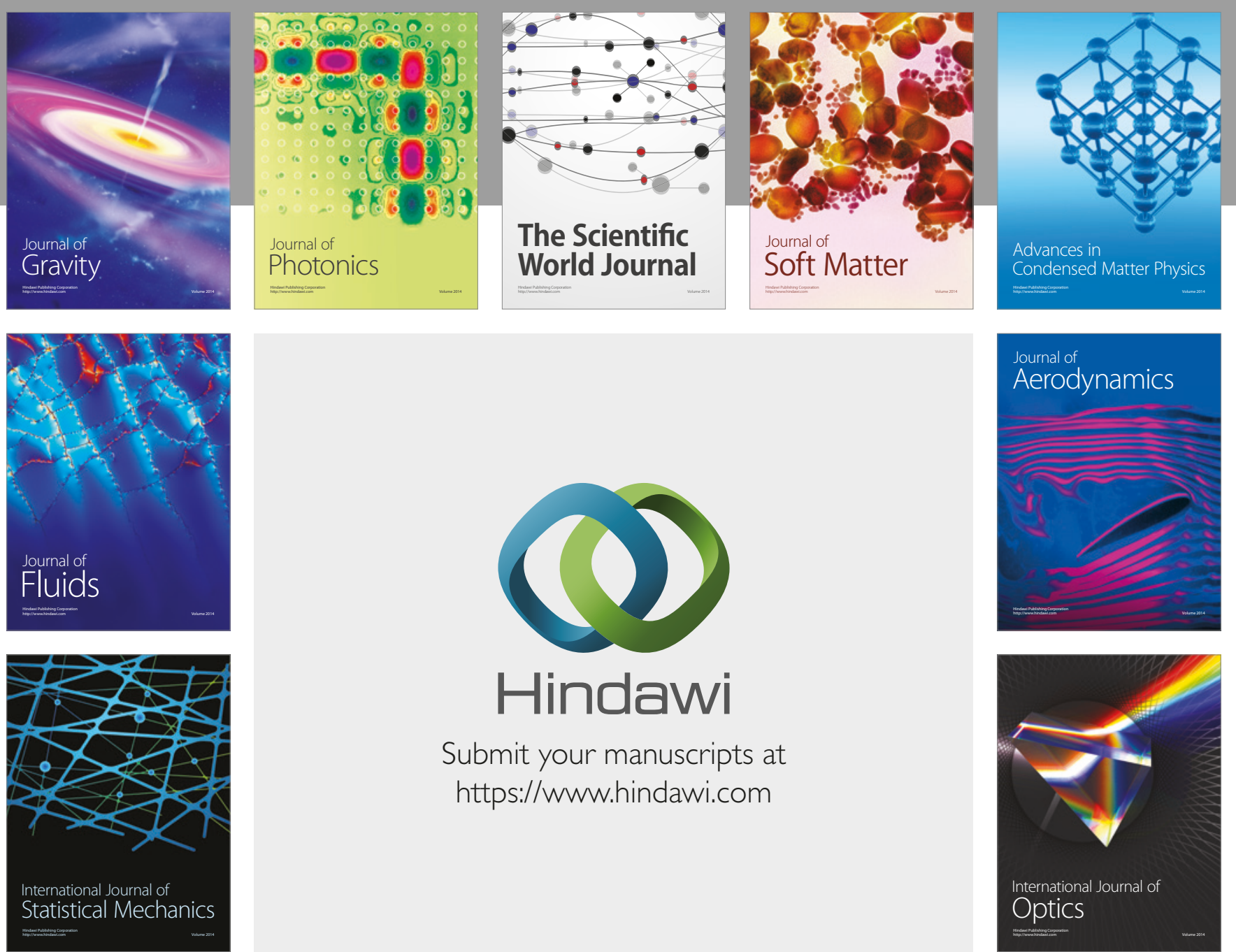

Submit your manuscripts at

https://www.hindawi.com
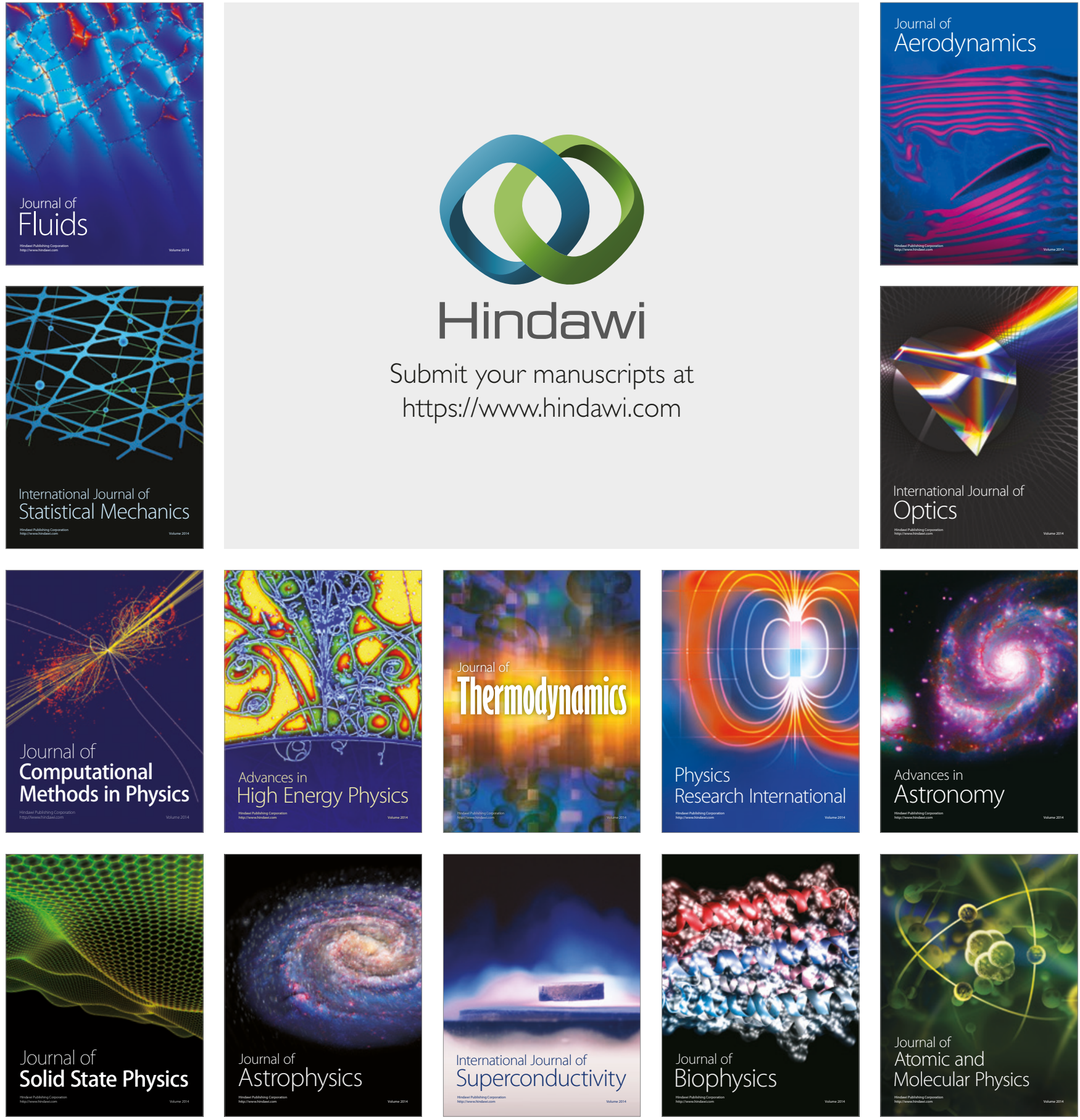